\title{
THE MOMENT ZETA FUNCTION AND APPLICATIONS
}

\author{
IGOR RIVIN
}

\begin{abstract}
Motivated by a probabilistic analysis of a simple game (itself inspired by a problem in computational learning theory) we introduce the moment zeta function of a probability distribution, and study in depth some asymptotic properties of the moment zeta function of those distributions supported in the interval $[0,1]$. One example of such zeta functions is Riemann's zeta function (which is the moment zeta function of the uniform distribution in $[0,1]$. For Riemann's zeta function we are able to show particularly sharp versions of our results.
\end{abstract}

\section{INTRODUCTION}

Consider the following setup: $(\Omega, \mu)$ is a space with a probability measure $\mu$, and $\omega_{1}, \ldots, \omega_{n}$ is a collection of measurable subsets of $\Omega$, with $\mu\left(\omega_{i}\right)=p_{i}$. We play a game as follows: The $j$ th step consists of picking a point $x_{j} \in \Omega$ at random, so that after $k$ steps we have the set $X_{k}=\left\{x_{1}, \ldots, x_{k}\right\}$. The game is considered to be over when

$$
\forall i \leq n, \quad X_{k} \cap \omega_{i} \neq X_{k} .
$$

We consider the duration of our game to be a random variable $T=$ $T\left(p_{1}, \ldots, p_{n}\right)$, and wish to compute the expection $E\left(p_{1}, \ldots, p_{n}\right)$. of $T$. This cannot, in general, be done without knowing the measures $p_{i_{1} i_{2} \ldots i_{k}}=\mu\left(\omega_{i_{1}} \cap \omega_{i_{2}} \cap \cdots \cap \omega_{i_{k}}\right)$, and in the sequel we will introduce the

\section{Independence Hypothesis:}

$$
p_{i_{1} i_{2} \ldots i_{k}}=p_{i_{1}} \times \cdots \times p_{i_{k}} .
$$

Estimates without using the independence hypothesis are shown in the companion paper [Rivin2002].

We now assume further that we don't actually know the measures $p_{1}, \ldots, p_{n}$, but know that they themselves are a sample from some

1991 Mathematics Subject Classification. 60E07, 60F15, 60J20, 91E40, 26C10.

Key words and phrases. learning theory, zeta functions, asymptotics.

The author would like to think the EPSRC and the NSF for support, and Natalia Komarova and Ilan Vardi for useful conversations. 
(known) probability distribution $\mathcal{F}$, of necessity supported in $[0,1]$. We consider $E\left(p_{1}, \ldots, p_{n}\right)$ as our random variable, and we wish to compute its expectation (over the space of all $n$-element samples from $\mathcal{F}$, and in particular we are interested in the limiting situation when $n$ is large.

Under the independence assumption, it turns out that we can write (Lemma 1.3):

$$
T=\sum_{\mathbf{s} \subseteq\{1, \ldots, n\}}(-1)^{|s|-1}\left(\frac{1}{1-p_{\mathbf{s}}}-1\right),
$$

where if $\mathbf{s}=\left\{i_{1}, \ldots, i_{k}\right\}$, we write $p_{\mathbf{s}}=p_{i_{1}} \times \cdots \times p_{i_{k}}$. To use equation (11) to understand the statistical behavior of $T$, we must introduce the moment zeta function of the probability distribution $\mathcal{F}$, defined as follows:

Definition A. Let $m_{k}=\int_{0}^{1} x^{k} d \mathcal{F}$ be the $k$-th moment of $\mathcal{F}$. Then

$$
\zeta_{\mathcal{F}}(s)=\sum_{k=1}^{\infty} m_{k}^{s}
$$

The sum in the definition above obviously converges only in some half-plane $\Re s>s_{0}$; the function can be analytically continued, but in the sequel we will be interested in asymptotic results for $s$ a large real number, so this will not use complex variable methods at all.

The relevance of this to our questions comes from Lemma 2.2, which we restate for convenience as:

Lemma B. Let $\mathcal{F}$ be a probability distribution as above, and let $x_{1}, \ldots, x_{n}$ be independent random variables with common distribution $\mathcal{F}$. Then

$$
\mathbb{E}\left(\frac{1}{1-x_{1} \ldots x_{n}}\right)=\zeta_{\mathcal{F}}(n) .
$$

In particular, the expectation is undefined whenever the zeta function is undefined.

Now, we can write (using Lemma B) the following formal identity:

$$
\mathbb{E}(T)=-\sum_{k=1}^{n}(-1)^{k}\left(\begin{array}{l}
n \\
k
\end{array}\right) \zeta_{\mathcal{F}}(k) .
$$

The identity is only formal, because $\zeta_{\mathcal{F}}(k)$ is not necessarily defined for all positive integers $k$. It is defined for all positive integers $k$ when $\mathcal{F}([1-x, 1]) \sim x^{\alpha}$, for $\alpha>1-$ this case is analyzed in Section 3 . If $\alpha=1$ (we will not deal with the case $\alpha<1$ in this paper; see Rivin2002), we write

$$
T=T_{1}-T^{\prime},
$$


where

$$
T_{1}=\sum_{i=1}^{n} \frac{1}{1-p_{i}} .
$$

$T_{1}$ has no expectation, but a $n$ goes to $\infty, T_{1} / n$ does converge in distribution to a stable law of exponent 1 (see [KR2001a and [KR2001b for many related results). The variable $T^{\prime}$ does possess a finite expectation, given by

$$
\mathbb{E}\left(T^{\prime}\right)=\sum_{k=2}^{n}(-1)^{k}\left(\begin{array}{l}
n \\
k
\end{array}\right) \zeta_{\mathcal{F}}(k) .
$$

The expressions given by Eq. (3) and Eq. (4) are analyzed in Sections 3 and 4 , and the following Theorems are shown:

Theorem C (Thm. 3.5). Let $\mathcal{F}$ be a continuous distribution supported on $[0,1]$, and let $f$ be the density of $\mathcal{F}$. Suppose further that

$$
\lim _{x \rightarrow 1} \frac{f(x)}{(1-x)^{\beta}}=c
$$

for $\beta, c>0$. Then,

$$
\begin{aligned}
& \lim _{n \rightarrow \infty} n^{-\frac{1}{1+\beta}}\left[\sum_{k=1}^{n}\left(\begin{array}{l}
n \\
k
\end{array}\right)(-1)^{k} \zeta_{\mathcal{F}}(k)\right] \\
=- & \int_{0}^{\infty} \frac{1-\exp \left(-c \Gamma(\beta+1) u^{1+\beta}\right)}{u^{2}} d u \\
= & -(c \Gamma(\beta+1))^{\frac{1}{\beta+1}} \Gamma\left(\frac{\beta}{\beta+1}\right) .
\end{aligned}
$$

and

Theorem D (Thm. 4.8). Let $\mathcal{F}$ be a continuous distribution supported on $[0,1]$, and let $f$ be the density of $\mathcal{F}$. Suppose further that

$$
\lim _{x \rightarrow 1} \frac{f(x)}{(1-x)}=c>0 \text {. }
$$

Then,

$$
\sum_{k=2}^{n}\left(\begin{array}{l}
n \\
k
\end{array}\right)(-1)^{k} \zeta_{\mathcal{F}}(k) \sim c n \log n .
$$

To get error estimates, we need stronger assumption on the function $f$ than the weakest possible assumption made in Theorem 4.8. The proof of the below follows by modifying slightly the proof of Lemma 4.7: 
Theorem E (Thm. 4.9). Let $\mathcal{F}$ be a continuous distribution supported on $[0,1]$, and let $f$ be the density of $\mathcal{F}$. Suppose further that

$$
f(x) \sim c(1-x)+O\left((1-x)^{\delta}\right),
$$

where $\delta>0$. Then,

$$
\sum_{k=2}^{n}\left(\begin{array}{l}
n \\
k
\end{array}\right)(-1)^{k} \zeta_{\mathcal{F}}(k) \sim c n \log n+O(n) .
$$

Our original probabilistic problem is thus completely resolved, but the sums given by Eqs. (3) and (国) are interesting in and of itself, and, with some more work (Section 5), we can considerably strengthen them as follows for the Riemann zeta function and its scaling:

Theorem F (Thm. 5.1).

$$
\sum_{k=2}^{n}\left(\begin{array}{l}
n \\
k
\end{array}\right)(-1)^{k} \zeta(k) \sim n \log n+(2 \gamma-1) n+O\left(\frac{1}{n}\right),
$$

where $\zeta$ is the Riemann zeta function and $\gamma$ is Euler's constant.

and

Theorem G (Thm. 5.2). Let $s>1$, then

$$
\sum_{k=1}^{n}\left(\begin{array}{l}
n \\
k
\end{array}\right)(-1)^{k} \zeta(s k) \sim \Gamma\left(1-\frac{1}{s}\right) n^{\frac{1}{s}} .
$$

It should be remarked that using the methods of section 5 higher order terms in the asymptotics can be obtained, if desired, but they seem to be of more limited interest.

\section{A FORMUla FOR THE WINNING Time $T$}

An application of the inclusion-exclusion principle gives us:

Lemma 1.1. The probability $l_{k}$ that we have won after $k$ steps is given by

$$
l_{k}=\prod_{i=1}^{n}\left(1-p_{i}^{k}\right)
$$

Note that the probability $s_{k}$ of winning the game on the $k$-th step is given by $s_{k}=l_{k}-l_{k-1}=\left(1-l_{k-1}\right)-\left(1-l_{k}\right)$. Since the expected number of steps $T$ is given by

$$
T=\sum_{k=1}^{\infty} k s_{k}
$$


we immediately have

$$
T=\sum_{k=1}^{\infty}\left(1-l_{k}\right)
$$

Lemma 1.2. The expected time $T$ of learning the concept $R_{0}$ is given by

$$
T=\sum_{k=1}^{\infty}\left(1-\prod_{i=1}^{n}\left(1-p_{i}^{k}\right)\right) .
$$

Since the sum above is absolutely convergent, we can expand the products and interchange the order of summation to get the following formula for $T$ :

Notation. Below, we identify subsets of $\{1, \ldots, n\}$ with multindexes (in the obvious way), and if $s=\left\{i_{1}, \ldots, i_{l}\right\}$, then

$$
p_{s} \stackrel{\text { def }}{=} p_{i_{1}} \cdots p_{i_{l}} \text {. }
$$

Lemma 1.3. The expression Eq. (5) can be rewritten as:

$$
T=\sum_{s \subseteq\{1, \ldots, n\}}(-1)^{|s|-1}\left(\frac{1}{1-p_{s}}-1\right),
$$

Proof. With notation as above,

$$
\prod_{i=1}^{m}\left(1-p_{i}^{k}\right)=\sum_{s \subseteq\{1, \ldots, n\}}(-1)^{|s|} p_{s}^{k},
$$

SO

$$
\begin{aligned}
T & =\sum_{k=1}^{\infty}\left(1-\prod_{i=1}^{n}\left(1-p_{i}^{k}\right)\right) \\
& =\sum_{k=1}^{\infty}\left(1-\sum_{s \subseteq\{1, \ldots, n\}}(-1)^{|s|} p_{s}^{k}\right) \\
& =\sum_{s \subseteq\{1, \ldots, n\}}(-1)^{|s|-1} \sum_{k=1}^{\infty} p_{s}^{k} \\
& =\sum_{s \subseteq\{1, \ldots, n\}}(-1)^{|s|-1}\left(\frac{1}{1-p_{s}}-1\right),
\end{aligned}
$$

where the change in the order of summation is permissible since all sums converge absolutely. 
Formula (6) is useful in and of itself, but we now use it to analyse the statistical properties of the time of success $T$ under our distribution and independence assumptions. For this we shall need to study the moment zeta function of a probability distribution, introduced below.

\section{Moment Zeta FUnCtion}

Definition 2.1. Let $\mathcal{F}$ be a probability distribution on a (possibly infinite) interval $I$, and let $m_{k}(\mathcal{F})=\int_{I} x^{k} \mathcal{F}(d x)$ be the $k$-th moment of $\mathcal{F}$. Then the moment zeta function of $\mathcal{F}$ is defined to be

$$
\zeta_{\mathcal{F}}(s)=\sum_{k=1}^{\infty} m_{k}^{s}(\mathcal{F}),
$$

whenever the sum is defined.

The definition is, in a way, motivated by the following:

Lemma 2.2. Let $\mathcal{F}$ be a probability distribution as above, and let $x_{1}, \ldots, x_{n}$ be independent random variables with common distribution $\mathcal{F}$. Then

$$
\mathbb{E}\left(\frac{1}{1-x_{1} \ldots x_{n}}\right)=\zeta_{\mathcal{F}}(n)
$$

In particular, the expectation is undefined whenever the zeta function is undefined.

Proof. Expand the fraction in a geometric series and apply Fubini's theorem.

Example 2.3. For $\mathcal{F}$ the uniform distribution on $[0,1], \zeta_{\mathcal{F}}$ is the familiar Riemann zeta function.

Our first observation is that for distributions supported in $[0,1]$, the asymptotics of the moments are determined by the local properties of the distribution at $x=1$. To show this, first recall that the Mellin transform of $f$ is defined to be

$$
\mathcal{M}(f)(s)=\int_{0}^{1} f(x) x^{s-1} d x
$$

Mellin transform is closely related to the Laplace transform. Making the substitution $x=\exp (-u)$, we see that

$$
\mathcal{M}(f)=\int_{0}^{\infty} f(\exp (-u)) \exp (-s u) d u
$$

so the Mellin transform of $f$ is equal to the Laplace transform of $f$ o exp, where $\circ$ denotes functional composition.

The following observation is both obvious and well-known: 
Lemma 2.4. $m_{k}(\mathcal{F})=\mathcal{M}(f)(k+1)$.

It follows that computing the asymptotic behavior of the $k$-th moment of $\mathcal{F}$ as a function of $k$ reduces to calculating the large $s$ asymptotics of the Mellin transform, which is tantamount to computing the asymptotics of the Laplace transform of $f \circ \exp$.

Theorem 2.5. Let $\mathcal{F}$ be a continuous distribution supported in $[0,1]$, let $f$ be the density of the distribution $\mathcal{F}$, and suppose that $f(1-x)=$ $c x^{\beta}+O\left(x^{\beta+\delta}\right)$, for some $\delta>0$. Then the $k$-th moment of $\mathcal{F}$ is asymptotic to $C k^{-(1+\beta)}$, for $C=c \Gamma(\beta+1)$.

Proof. The asymptotics of the Laplace transform are easily computed by Laplace's method, and in the case we are interested in, Watson's lemma (see, eg, BenOrsa ) tells us that if $f(x) \asymp c(1-x)^{\beta}$, then $\mathcal{M}(f)(s) \asymp c \Gamma(\beta+1) x^{-(\beta+1)}$.

Corollary 2.6. Under the assumptions of Theorem 2.5, $\zeta_{\mathcal{F}}(s)$ is defined for $s>1 /(1+\beta)$.

We will need another observation:

Lemma 2.7. For $\mathcal{F}$ supported in $[0,1], m_{k}(\mathcal{F})$ is monotonically decreasing as a function of $k$.

Proof. Immediate.

Below we shall analyze three cases. In the sequel, we set $\alpha=\beta+1$.

$$
\text { 3. } \alpha>1
$$

In this case, we use our assumptions to rewrite Eq. (6) as

$$
\mathbb{E}(T)=-\sum_{k=1}^{n}\left(\begin{array}{l}
n \\
k
\end{array}\right)(-1)^{k} \zeta_{\mathcal{F}}(k)
$$

This, in turn, can be rewritten (by expanding the definition of zeta) as

$$
\mathbb{E}(T)=-\sum_{j=1}^{\infty}\left[\left(1-m_{j}(\mathcal{F})\right)^{n}-1\right]=\sum_{j=1}^{\infty}\left[1-\left(1-m_{j}(\mathcal{F})\right)^{n}\right]
$$

Since the terms in the sum is monotonically decreasing (as a function of $j$ ) by Lemma 2.7, the sum in Eq. (9) can be approximated by an integral of any monotonic interpolation $m$ of the sequence $m_{j}(\mathcal{F})$ we will interpolate by $m(x)=\mathcal{M}(f)(x+1))$. The error of such an 
approximation is bounded by the first term, which, in turn, isbounded in absolute value by 2 , to get

$$
T=-\int_{1}^{\infty}\left[(1-m(x))^{n}-1\right] d x+O(1)
$$

where the error term is bounded above by 2 . We shall write

$$
T_{0}=-\int_{1}^{\infty}\left[(1-m(x))^{n}-1\right] d x
$$

Now, let us assume that

$$
\lim _{x \rightarrow \infty} x^{\alpha} m(x)=L,
$$

for some $\alpha>1$. We substitute $x=n^{1 / \alpha} / u$, to get

$$
T_{0}=n^{\frac{1}{\alpha}} \int_{0}^{n^{\frac{1}{\alpha}}} \frac{\left[1-\left(1-m\left(n^{1 / \alpha} / u\right)\right)^{n}\right]}{u^{2}} d u=n^{\frac{1}{\alpha}}\left[I_{1}(n)+I_{2}(n)\right],
$$

where

$$
I_{1}(n)=\int_{0}^{n^{\frac{1}{3 \alpha}}} \frac{\left[1-\left(1-m\left(n^{1 / \alpha} / u\right)^{n}\right]\right.}{u^{2}} d u
$$

and

$$
I_{2}(n)=\int_{n \frac{1}{3 \alpha}}^{n^{\frac{1}{\alpha}}} \frac{\left[1-\left(1-m\left(n^{1 / \alpha} / u\right)^{n}\right]\right.}{u^{2}} d u .
$$

We will need the following:

Lemma 3.1. Let $f_{n}(x)=(1-x / n)^{n}$, and let $0 \leq z<1 / 2$.

$$
f_{n}(x)=\exp (-x)\left[1-\frac{x^{2}}{2 n}+O\left(\frac{x^{3}}{n^{2}}\right)\right] \text {. }
$$

Proof. Note that

$$
\log f_{n}(x)=n \log (1-x / n)=-x-\sum_{k=2}^{\infty} \frac{x^{k}}{k n^{k-1}} .
$$

The assertion of the lemma follows by exponentiating the two sides of the above equation.

Now:

\section{Lemma 3.2.}

$$
\lim _{n \rightarrow \infty} n^{\frac{1}{\alpha}} I_{2}(n)=0
$$


Proof. The integrand of $I_{2}(n)$ is monotonically decreasing, and so

$$
I_{2}(n) \leq n^{-\frac{2}{3 \alpha}}\left[1-\left(1-m\left(n^{\frac{2}{3 \alpha}}\right)\right)^{n}\right] .
$$

By our assumption Eq. (11) and by Lemma 3.1 we see that the right hand side goes to zero (exponentially fast).

\section{Lemma 3.3.}

$$
\lim _{n \rightarrow \infty} I_{1}(n)=\int_{0}^{\infty} \frac{1-\exp \left(-L u^{\alpha}\right)}{u^{2}} d u
$$

Proof. Immediate from Eq. (11) and Lemma 3.1. Note that the integral converges when $\alpha$ is greater than 1 .

\section{Remark 3.4.}

$$
\int_{0}^{\infty} \frac{1-\exp \left(-L u^{\alpha}\right)}{u^{2}} d u=L^{\frac{1}{\alpha}} \Gamma\left(\frac{\alpha-1}{\alpha}\right) .
$$

Proof.

$$
\int_{0}^{\infty} \frac{1-\exp \left(-L u^{\alpha}\right)}{u^{2}} d u=\lim _{\epsilon \rightarrow 0}\left[\frac{1}{\epsilon}-\int_{\epsilon}^{\infty} \frac{\exp \left(-L u^{\alpha}\right)}{u^{2}} d u\right] .
$$

To prove the remark we need to analyze the behavior of the integral above as $\epsilon \rightarrow 0$. First, we change variables: $v=L u^{\alpha}$. Then,

$$
\int_{\epsilon}^{\infty} \frac{\exp \left(-L u^{\alpha}\right)}{u^{2}} d u=\frac{L^{1 / \alpha}}{\alpha} \int_{L \epsilon^{\alpha}}^{\infty} \exp (-v) v^{-(1+1 / \alpha)} d v .
$$

Integrating by parts, get

$$
\int_{L \epsilon^{\alpha}}^{\infty} \exp (-v) v^{-(1+1 / \alpha)} d v=-\left.\alpha \exp (-v) v^{1 / \alpha}\right|_{L \epsilon^{\alpha}} ^{\infty}-\alpha \int_{L \epsilon^{\alpha}} \exp (-v) v^{-1 / \alpha}
$$

Since $1 / \alpha<1, \int_{0}^{\infty} \exp (-v) v^{-1 / \alpha} d v=\Gamma(1-1 / \alpha)$, from which the assertion of the remark follows.

We summarize as follows:

Theorem 3.5. Let $\mathcal{F}$ be a continuous distribution supported on $[0,1]$, and let $f$ be the density of $\mathcal{F}$. Suppose further that

$$
\lim _{x \rightarrow 1} \frac{f(x)}{(1-x)^{\beta}}=c,
$$


for $\beta, c>0$. Then,

$$
\begin{aligned}
& \lim _{n \rightarrow \infty} n^{-\frac{1}{1+\beta}}\left[\sum_{k=1}^{n}\left(\begin{array}{l}
n \\
k
\end{array}\right)(-1)^{k} \zeta_{\mathcal{F}}(k)\right] \\
=- & \int_{0}^{\infty} \frac{1-\exp \left(-c \Gamma(\beta+1) u^{1+\beta}\right)}{u^{2}} d u \\
= & -(c \Gamma(\beta+1))^{\frac{1}{\beta+1}} \Gamma\left(\frac{\beta}{\beta+1}\right) .
\end{aligned}
$$

Proof. Follows from Lemmas 3.3 and 3.2 together with theorem 2.5 and Remark 3.4.

$$
\text { 4. } \alpha=1
$$

In this case,

$$
f(x)=L+o(1)
$$

as $x$ approaches 1 , and so Theorem 2.5 tells us that

$$
\lim _{j \rightarrow \infty} j m_{j}(\mathcal{F})=L \text {. }
$$

It is not hard to see that $\zeta_{\mathcal{F}}(n)$ is defined for $n \geq 2$. We break up the expression in Eq. (6) as

$$
T=\sum_{j=1}^{n} \frac{1}{1-p_{j}}-1+\sum_{s \subseteq\{1, \ldots, n\},}(-1)^{|s|-1}\left(\frac{1}{1-p_{s}}-1\right) .
$$

Let

$$
\begin{gathered}
T_{1}=\sum_{j=1}^{n} \frac{1}{1-p_{j}}-1, \\
T_{2}=\sum_{s \subseteq\{1, \ldots, n\}, \quad}(-1)^{|s|-1}\left(\frac{1}{1-p_{s}}-1\right) .
\end{gathered}
$$

The first sum $T_{1}$ has no expectation, however $T_{1} / n$ does have have a stable distribution centered on $c \log n+c_{2}$. We will keep this in mind, but now let us look at the second sum $T_{2}$. It can be rewritten as

$$
T_{2}(n)=-\sum_{j=1}^{\infty}\left[\left(1-m_{j}(\mathcal{F})\right)^{n}-1+n m_{j}(\mathcal{F})\right]
$$

Lemma 4.1. The quantity $y_{j}=\left(1-m_{j}(\mathcal{F})\right)^{n}-1+n m_{j}(\mathcal{F})$ is a monotonic function of $j$. 
Proof. We know that $m_{j}(\mathcal{F})$ is a monotonically decreasing positive function of $j$, and that $m_{0}(\mathcal{F})=1$. It is sufficient to show that the function $g_{n}(x)=(1-x)^{n}+n x$ is monotonic for $x \in(0,1]$. We compute

$$
\frac{d g_{n}(x)}{d x}=n\left(1-(1-x)^{n-1}\right)>0
$$

for $x \in(0,1)$.

Lemma 4.1 allows us to use the same method as in section 3 under the assumption that the $k$-th moment is asymptotic to $k^{\alpha}$ (this time for $\alpha \leq 1)$. Since the term $y_{j}$ is bounded above by a constant times $n$, we can write

$$
T_{2}(n)=S_{2}(n)+O(n)
$$

where

$$
S_{2}(n)=n \int_{0}^{n} \frac{\left[1-n m(n / u)-\left(1-m(n / u)^{n}\right]\right.}{u^{2}} d u \text {. }
$$

Remark 4.2. The error term in Eq. (10) above can be improved in the case where $\mathcal{F}$ is the uniform distribution on $[0,1]$, in which case $m_{j}=1 / j$. In that case $T_{2}(n)=S_{2}(n)-\gamma n+O(1)$, where $\gamma$ is Euler's constant.

Proof. In this case, we write

$$
\begin{array}{r}
T_{2}(n)=\lim _{k \rightarrow \infty}-\sum_{j=1}^{k}\left[\left(1-m_{j}(\mathcal{F})\right)^{n}-1+n m_{j}(\mathcal{F})\right] \\
=-\sum_{j=1}^{k}\left[\left(1-m_{j}(\mathcal{F})\right)^{n}-1\right]-n \sum_{j=1}^{k} m_{j} .
\end{array}
$$

The terms in the first sum are decreasing, so the first sum can be approximated by an integral with total error $O(1)$. As for the second sum, since $m_{j}=1 / j$, it is well-known (eg, Euler-Maclaurin summation) that

$$
\sum_{j=1}^{k} \frac{1}{j}=\int_{1}^{k} \frac{d x}{x}+\gamma+O\left(\frac{1}{k}\right)
$$

from which the assertion of the remark follows.

To understand the asymptotic behavior of $S_{2}(n)$ we write

$$
S_{2}(n)=n\left[I_{1}(n)+I_{2}(n)+I_{3}(n)+I_{4}(n)\right],
$$


where

$$
\begin{gathered}
I_{1}(n)=\int_{0}^{1} \frac{\left[1-n m(n / u)-\left(1-m\left(\frac{n}{u}\right)\right)^{n}\right]}{u^{2}} d u, \\
I_{2}(n)=\int_{1}^{n^{\frac{1}{3}}} \frac{\left[1-\left(1-m\left(\frac{n}{u}\right)\right)^{n}\right]}{u^{2}} d u, \\
I_{3}(n)=\int_{n^{\frac{1}{3}}}^{n} \frac{\left[1-\left(1-m\left(\frac{n}{u}\right)\right)^{n}\right]}{u^{2}} d u, \\
I_{4}(n)=-n \int_{1}^{n} \frac{m(n / u)}{u^{2}} d u .
\end{gathered}
$$

\section{Lemma 4.3.}

$$
\lim _{n \rightarrow \infty} I_{1}(n)=\int_{0}^{1} \frac{1-\exp (-L u)-L u}{u^{2}} d u
$$

Proof. Immediate from the estimate Eq. (13) and Lemma 3.1.

\section{Lemma 4.4.}

$$
\lim _{n \rightarrow \infty} I_{2}(n)=\int_{1}^{\infty} \frac{1-\exp (-L u)}{u^{2}} d u
$$

Proof. Again, immediate from Eq. (13) and Lemma 3.1.

\section{Remark 4.5.}

$$
\int_{0}^{1} \frac{1-\exp (-L u)-L u}{u^{2}}+\int_{1}^{\infty} \frac{1-\exp (-L u)}{u^{2}}=L(1-\gamma-\log L),
$$

where $\gamma$ is Euler's constant.

Proof.

$$
\begin{array}{r}
\int_{0}^{1} \frac{1-\exp (-L u)-L u}{u^{2}} d u+\int_{1}^{\infty} \frac{1-\exp (-L u)}{u^{2}} d u= \\
\lim _{\epsilon \rightarrow 0}\left\{-\int_{\epsilon}^{\infty}\left[\frac{\exp (-L u)}{u^{2}}+\frac{1}{u^{2}}\right] d u-L \int_{\epsilon}^{1} \frac{d u}{u}\right\}= \\
\lim _{\epsilon \rightarrow 0}\left\{\frac{1}{\epsilon}+L \log \epsilon-\int_{\epsilon}^{\infty} \frac{\exp (-L u)}{u^{2}} d u\right\} .
\end{array}
$$


To evaluate the last limit, we need to compute the expansion as $\epsilon \rightarrow 0$ of the last integral. Changing variables $v=L u$, we get

$$
\begin{array}{r}
\int_{\epsilon}^{\infty} \frac{\exp (-L u)}{u^{2}} d u=L \int_{L \epsilon}^{\infty} \frac{\exp (-v)}{v^{2}} d v= \\
L\left[-\left.\frac{\exp (-v)}{v}\right|_{L \epsilon} ^{\infty}-\int_{L \epsilon}^{\infty} \frac{\exp (-v)}{v} d v\right]= \\
L\left[\frac{\exp (-v)}{L \epsilon}-\left.\exp (-v) \log (v)\right|_{L \epsilon} ^{\infty}-\int_{L \epsilon}^{\infty} \exp (-v) \log (v) d v\right]= \\
\frac{\exp (-L \epsilon)}{\epsilon}+L \exp (-L \epsilon) \log (\epsilon)+ \\
L \log L \exp (-L \epsilon)-L \int_{L \epsilon}^{\infty} \exp (-v) \log (v) d v .
\end{array}
$$

Substituting into Eq. (22), we get

$$
\begin{array}{r}
\int_{0}^{1} \frac{1-\exp (-L u)-L u}{u^{2}} d u+\int_{1}^{\infty} \frac{1-\exp (-L u)}{u^{2}} d u= \\
\lim _{\epsilon \rightarrow 0}\left\{\frac{1-\exp (-L \epsilon)}{\epsilon}+L(1-\exp (-L \epsilon)) \log \epsilon\right. \\
\left.-L \log L \exp (-L \epsilon)+\int_{L \epsilon}^{\infty} \exp (-v) \log v d v\right\} \\
=L\left(1-\log L+\int_{L \epsilon}^{\infty} \exp (-v) \log v d v\right) .
\end{array}
$$

Since $\int_{0}^{\infty} \log (x) \exp (-x) d x=-\gamma$, the result follows.

\section{Lemma 4.6.}

$$
\lim _{n \rightarrow \infty} n I_{3}(n)=0
$$

Proof. See the proof of Lemma 3.2 .

\section{Lemma 4.7.}

$$
\lim _{n \rightarrow \infty}-\frac{I_{4}(n)}{\log n}=L .
$$

Proof. We shall show that the limit in question lies between $(1-\epsilon) L$ and $(1+\epsilon) L$, for any $\epsilon>0$, from which the conclusion of the lemma obviously follows. To do that, pick $C$, such that

$$
1-\epsilon / 4 \leq x m(x) \leq 1+\epsilon / 4
$$

for $x>C$. Now, write

$$
\int_{1}^{n} \frac{m(n / u)}{u^{2}} d u=J_{1}(n)+J_{2}(n),
$$


where

$$
\begin{aligned}
& J_{1}(n)=\int_{1}^{\frac{n}{C}} \frac{m(n / u)}{u^{2}} d u \\
& J_{2}(n)=\int_{\frac{n}{C}}^{n} \frac{m(n / u)}{u^{2}} d u .
\end{aligned}
$$

Observe that

$$
0<J_{2}(n)=\frac{1}{n} \int_{1}^{C} m(x) d x \leq \frac{C-1}{n}
$$

while

SO

$$
\frac{1-\epsilon / 4}{n} \int_{1}^{\frac{n}{C}} \frac{d u}{u} \leq J_{1}(n) \leq \frac{1+\epsilon / 4}{n} \int_{1}^{\frac{n}{C}} \frac{d u}{u}
$$

$$
\frac{(1-\epsilon / 4)}{n}(\log n-\log C) \leq J_{1}(n) \leq \frac{(1+\epsilon / 4)}{n}(\log n-\log C) .
$$

If we now pick $N=C^{4 / \epsilon}$, it is clear that for $n>N$,

$$
(1-\epsilon / 2) \log n \leq J_{1}(n) \leq(1+\epsilon / 2) \log n,
$$

while $J_{2}$ is bounded above in absolute value by $C^{1-4 / \epsilon}$.

The above lemmas can be summarized in the following

Theorem 4.8. Let $\mathcal{F}$ be a continuous distribution supported on $[0,1]$, and let $f$ be the density of $\mathcal{F}$. Suppose further that

$$
\lim _{x \rightarrow 1} \frac{f(x)}{(1-x)}=c>0 \text {. }
$$

Then,

$$
\sum_{k=2}^{n}\left(\begin{array}{l}
n \\
k
\end{array}\right)(-1)^{k} \zeta_{\mathcal{F}}(k) \sim c n \log n .
$$

To get error estimates, we need stronger assumption on the function $f$ than the weakest possible assumption made in Theorem 4.8. The proof of the below follows by modifying slightly the proof of Lemma 4.7:

Theorem 4.9. Let $\mathcal{F}$ be a continuous distribution supported on $[0,1]$, and let $f$ be the density of $\mathcal{F}$. Suppose further that

$$
f(x) \sim c(1-x)+O\left((1-x)^{\delta}\right),
$$

where $\delta>0$. Then,

$$
\sum_{k=2}^{n}\left(\begin{array}{l}
n \\
k
\end{array}\right)(-1)^{k} \zeta_{\mathcal{F}}(k) \sim c n \log n+O(n) .
$$




\section{RIEMANN ZETA FUnCTION}

The proof of the key Lemma 4.7 is trivial in the case where $f(x)=1$, and so $\zeta_{\mathcal{F}}$ is the Riemann zeta function. In that case, however, we get the following much stronger result:

\section{Theorem 5.1.}

$$
\sum_{k=2}^{n}\left(\begin{array}{l}
n \\
k
\end{array}\right)(-1)^{k} \zeta(k) \sim n \log n+(2 \gamma-1) n+O\left(\frac{1}{n}\right),
$$

where $\zeta$ is the Riemann zeta function and $\gamma$ is Euler's constant.

It should also be noted that the results of Section 3 immediately imply the following:

Theorem 5.2. Let $s>1$, then

$$
\sum_{k=1}^{n}\left(\begin{array}{l}
n \\
k
\end{array}\right)(-1)^{k} \zeta(s k) \sim \Gamma\left(1-\frac{1}{s}\right) n^{\frac{1}{s}} .
$$

To prove Theorem 5.1 we need to sharpen some of the estimates of the preceding section. First:

Lemma 5.3. Let the notation be as in the preceding section. When $m(x)=\frac{1}{x}$,

$$
\begin{gathered}
I_{1}(n)=\int_{0}^{1} \frac{1-\exp (-u)-u}{u^{2}} d u+\frac{1}{2 n} \int_{0}^{1} \exp (-u) d u+O\left(\frac{1}{n^{2}}\right), \\
I_{2}(n)=\int_{1}^{\infty} \frac{1-\exp (-u)}{u^{2}} d u+\frac{1}{2 n} \int_{1}^{\infty} \exp (-u) d u .
\end{gathered}
$$

Proof. Imediate from the expansion in Lemma 3.1.

We can also sharpen the statement of Lemma 4.6:

\section{Lemma 5.4.}

$$
\lim _{n \rightarrow \infty} n^{k} I_{3}(n)=0
$$

for any $k$.

Proof. This statement holds in general, and no change in argument is necessary.

In the case where $m(x)=1 / x$, Lemma 4.7 is immediate, and has no error term:

\section{Lemma 5.5.}

$$
I_{4}(n)=-\log (n)
$$


Proof. Immediate.

We now have:

\section{Theorem 5.6.}

$$
\sum_{k=2}^{n}\left(\begin{array}{l}
n \\
k
\end{array}\right)(-1)^{k} \zeta(k) \sim n \log n+(2 \gamma-1) n+O(1),
$$

Proof. Lemmas 5.3, 5.4 and 5.5, combined with Remark 4.2.

To improve the error term from that in Theorem 5.6, it is necessary to sharpen the estimate in Remark 1.2 to:

Theorem 5.7. With the notation of Remark 4.9,

$$
T_{2}(n)=S_{2}(n)-\gamma n-\frac{1}{2}+O\left(\frac{1}{n}\right) .
$$

Proof. The Theorem will follow immediately from Lemma 5.8 and the results of Section 5.1.

\section{Lemma 5.8.}

$$
\lim _{N \rightarrow \infty} \sum_{j=1}^{N} \frac{1}{j}-\log (n)=\gamma .
$$

Proof. Well-known.

5.1. A sum and an integral. Let

$$
\begin{gathered}
S_{n}(N)=\sum_{j=1}^{N}\left(1-\frac{1}{j}\right)^{n}, \\
I_{n}(N)=\int_{1}^{N}\left(1-\frac{1}{x}\right)^{n} d x, \\
D_{n}(N)=S_{n}(N)-I_{n}(N), \\
D_{n}=\lim _{N \rightarrow \infty} D_{n}(N) .
\end{gathered}
$$

In this section we shall prove the following result:

\section{Theorem 5.9.}

$$
D_{n}=\frac{1}{2}+o\left(\frac{1}{n}\right) .
$$

We will need the following preliminary results: 
Lemma 5.10. Let $f$ be a $C^{1}$ function defined on $[0, \infty)$. Then

$$
\sum_{k=0}^{N} f(k)=\frac{1}{2}[f(0)+f(N)]+\int_{0}^{N} f(t) d t+\int_{0}^{N}\left(\{t\}-\frac{1}{2}\right) f^{\prime}(t) d t .
$$

Proof. Integration by parts - see Exercises for Section 6.7 of [BenOrsz.

Lemma 5.11. Let $f$ be a $C^{2}$ function defined on $[0, \infty)$, such that $f^{\prime \prime}$ is bounded, and $f^{\prime \prime}(x)=O\left(1 / x^{2}\right)$. Then

$$
\left|\sum_{k=0}^{\infty} \int_{\frac{k}{n}}^{\frac{k+1}{n}}\left(x-\frac{k+\frac{1}{2}}{n}\right) f(x) d x-\frac{1}{4 n^{3}} \sum_{k=0}^{\infty} f^{\prime}\left(\frac{k+\frac{1}{2}}{n}\right)\right|=O\left(\frac{1}{n^{4}}\right) .
$$

Proof. On the interval $[k / n,(k+1) / n]$ we can write

$$
f(x)=f\left(\frac{k+\frac{1}{2}}{n}\right)+f^{\prime}\left(\frac{k+\frac{1}{2}}{n}\right)\left(x-\frac{k+\frac{1}{2}}{n}\right)+R_{2}(x),
$$

where, by Taylor's theorem, $\left|R_{2}(x)\right| \leq x^{2} \max _{x \in[k / n,(k+1) / n]} f^{\prime \prime}(x)$. The assertion of the lemma then follows by integration of Eq. (27).

Lemma 5.12. Under the assumptions of Lemma 5.11, together with the assumtption that $f$ and all of its derivatives vanish at 0

$$
\left|\sum_{k=0}^{\infty} f^{\prime}\left(\frac{k+\frac{1}{2}}{n}\right)\right|=O\left(\frac{1}{n}\right) .
$$

Proof. Let $g(y)=f^{\prime}((x+1 / 2) / n)$. Then:

$$
\begin{aligned}
\sum_{k=0}^{\infty} f^{\prime}\left(\frac{k+\frac{1}{2}}{n}\right) & =\sum_{k=0}^{\infty} g(k) \\
& =\frac{1}{2} g(0)+\int_{0}^{\infty} g(x) d x+\int_{0}^{\infty}\left(\{x\}-\frac{1}{2}\right) g^{\prime}(x) d x \\
& =\frac{1}{2} f^{\prime}\left(\frac{1}{2 n}\right)+\int_{0}^{\infty} f^{\prime}\left(\frac{x+\frac{1}{2}}{n}\right) d x+O\left(\frac{1}{n}\right) \\
& =n \int_{\frac{1}{2 n}}^{\infty} f^{\prime}(x) d x+O\left(\frac{1}{n}\right) \\
& =O\left(\frac{1}{n}\right)
\end{aligned}
$$


Now we proceed to the proof of Theorem 5.9 per se. First:

\section{Lemma 5.13.}

$$
D_{n}=\frac{1}{2}+n \int_{1}^{\infty} \frac{\left(\{x\}-\frac{1}{2}\right)\left(1-\frac{1}{x}\right)^{n-1}}{x^{2}} d x .
$$

Proof. Immediate corollary of Lemma 5.10 .

Proof of Theorem 5.9. By Lemma 5.13 it remains to analyse the asymptotic behavior of

$$
J_{n}=(n+1) \int_{1}^{\infty} \frac{\left(\{x\}-\frac{1}{2}\right)\left(1-\frac{1}{x}\right)^{n}}{x^{2}} d x .
$$

(the expression occuring in Lemma 5.13 is actually $J_{n-1}$, we have changed the variable for notational convenience). First, we make the substitution $x=n y$, to get

$$
J_{n}=\frac{(n+1)}{n} \underbrace{\int_{\frac{1}{n}}^{\infty} \frac{\left(\{n y\}-\frac{1}{2}\right)\left(1-\frac{1}{n y}\right)^{n}}{y^{2}} d x}_{K_{n}}
$$

where clearly $J_{n} \sim K_{n}$. We now write

$$
K_{n}=[\underbrace{\int_{\frac{1}{n}}^{n^{-\frac{1}{3}}}}_{K_{n}^{\prime}}+\underbrace{\int_{n^{-\frac{1}{3}}}^{\infty}}_{K_{n}^{\prime \prime}}] \frac{\left(\{n y\}-\frac{1}{2}\right)\left(1-\frac{1}{n y}\right)^{n}}{y^{2}} d x .
$$

The integrand of $K_{n}^{\prime}$ is bounded above by

$$
\left(1-n^{-\frac{2}{3}}\right)^{n}
$$

while the interval of integration is polynomial in length, which implies that $K_{n}^{\prime}$ decreases faster than any power of $n$, and so can be ignored for our purposes. On the other hand, Lemma 3.1 implies that

$$
\begin{aligned}
K_{n}^{\prime \prime} & \sim \int_{0}^{\infty}\left(\{n y\}-\frac{1}{2}\right) \frac{\exp \left(-\frac{1}{y}\right)}{y^{2}} d y \\
& =\sum_{k=0}^{\infty} \int_{\frac{k}{n}}^{\frac{k+1}{n}}\left[n y-\frac{1}{2}-k\right] \frac{\exp \left(-\frac{1}{y}\right)}{y^{2}} d y \\
& =n \sum_{k=0}^{\infty} \int_{\frac{k}{n}}^{\frac{k+1}{n}}\left[y-\frac{k+\frac{1}{2}}{n}\right] \frac{\exp \left(-\frac{1}{y}\right)}{y^{2}} d y .
\end{aligned}
$$


We can now apply Lemmas 5.11 and 5.12 with

$$
f(x)=\frac{\exp \left(-\frac{1}{y}\right)}{y^{2}} ;
$$

it is easy to check that $f(x)$ satisfies the assumptions. Theorem 5.9 follows.

\section{REFERENCES}

[BenOrsz] C. M. Bender and S. Orszag (1999) Advanced mathematical methods for scientists and engineers, I, Springer-Verlag, New York.

[KR2001a] Komarova, N. L. and Rivin, I. (2001) Harmonic mean, random polynomials and stochastic matrices, preprint.

[KR2001b] Komarova, N. L. and Rivin, I. (2001) On the mathematics of learning.

[Rivin2002] Igor Rivin. (2002) An analysis of the batch learning algorithm.

Mathematics department, University of Manchester, Oxford Road, MANCHESTER M13 9PL, UK

Mathematics Department, Temple University, Philadelphia, PA 19122

Mathematics Department, Princeton University, Princeton, NJ 08544

E-mail address: irivin@math.princeton.edu 